\title{
Peripheral possibilities: revealing originality and encouraging dialogue through a reconsideration of 'marginal' IR scholarship
}

\author{
Helen Louise Turton and Lucas G. Freire
}

Journal of International Relations and Development (2017) 20, 458. doi:10.1057/jird.2015.17; advance online publication, 17 April 2015

Correction to: Journal of International Relations and Development advance online publication, 21 November 2014; doi: 10.1057/jird.2014.24

In 'About the Authors' on page 24 of the article the author's name was misspelled. The correct spelling should have appeared as Lucas G. Freire.

We apologise for this oversight. The content and research of the article remains unchanged. 